\title{
ÍNDICE RELATIVO DE MODERNIZAÇÃo AGRÍCOLA NA REGIÃO NORTE ${ }^{1}$
}

\author{
Rubicleis Gomes da Silva ${ }^{2}$ \\ Elaine Aparecida Fernandes ${ }^{3}$
}

Resumo - A atividade agrícola possui expressiva relevância para a agricultura da região Norte. Em relação à modernização agrícola, as pesquisas são conduzidas de forma agregada (nacional), o que impossibilita verificar, efetivamente, qual o grau de desenvolvimento municipal e quais as variáveis que impactam este desenvolvimento. De forma geral, pretende-se determinar o grau de modernização agrícola nos municípios do Norte. Observa-se que esta região apresenta baixos níveis estaduais de modernização, os quais se justificam pela recente expansão da fronteira agrícola, pelo baixo nível de investimentos governamentais, pela falta de políticas públicas destinadas ao desenvolvimento agrícola e, por fim, pelos movimentos ambientalistas.

Palavras-chave: Modernização agrícola, região Norte e análise fatorial.

\section{Introdução}

A região Norte do Brasil, especialmente a Amazônia, é freqüentemente lembrada em discussões referentes ao meio ambiente. Com isso, a agricultura fica relegada a segundo plano, embora essa atividade seja importante tanto para gerar empregos como para promover o desenvolvimento econômico e regional.

A atividade agrícola é de suma importância para a economia da região Norte, no entanto, não existem estudos que priorizem seu tratamento.

\footnotetext{
Recebido em 30/12/2004. Aceito em 10/02/2005. Os autores agradecem à CAPES, ao CNPq e à Universidade Federal do Acre (UFAC), pelo financiamento da pesquisa.

2 Bolsista CAPES/PQI/UFAC, economista, servidor da UFAC, Mestre em Economia Aplicada e Doutorando em Economia pelo Departamento de Economia Rural (DER) da Universidade Federal de Viçosa (UFV). E-mail: rubicleis@uol.com.br

3 Bolsista CNPq, economista, Mestre em Economia Aplicada e Doutoranda em Economia Aplicada pelo Departamento de Economia Rural (DER) da Universidade Federal de Viçosa (UFV). E-mail: elainef1@vicosa.ufv.br
} 
Em relação à modernização agrícola, as pesquisas são conduzidas de forma agregada (nacional), o que impossibilita verificar, efetivamente, qual o grau de desenvolvimento municipal. Assim, a falta de pesquisas e o problema da modernização agrícola motivam este estudo, cuja relevância reside em fornecer um diagnóstico sobre o grau de modernização dos municípios da região Norte do país.

De forma geral, busca-se determinar o grau de modernização agrícola dos municípios do Norte. Especificamente, pretende-se: a) quantificar o Indicador Parcial de Modernização Agrícola - IPMA; b) construir o Índice de Modernização Agrícola (IRMA) da região Norte do Brasil.

Vários estudos no Brasil detiveram-se na questão do desenvolvimento agrícola, em particular, da modernização. Dentre eles, os de Hoffman (1986), que determinou o grau de modernização agrícola de 157 microrregiões brasileiras; de Ferreira Jr. e Baptista (2004), que estudaram a modernização das microrregiões do estado de Minas Gerais; e de Souza e Lima (2003), que analisaram a intensidade e a dinâmica da modernização agrícola no Brasil.

A construção do IRMA, conjuntamente com a análise por estado do Norte, constitui a inovação presente neste trabalho. Os estudos anteriores relacionados com modernização elaboravam uma hierarquização em que apenas constatações de que o ordenamento do tipo "o município A é mais moderno que B" podia ser feito. Contudo, a determinação do quanto ele era mais moderno não era considerada nas análises. É válido mencionar que a aplicação da metodologia utilizada neste artigo não encontra nenhuma referência na área de modernização agrícola. Os trabalhos que utilizaram essa metodologia visaram determinar o nível de degradação ambiental de municípios ou microrregiões, a exemplo dos trabalhos pioneiros de Lemos (2001), Silva e Ribeiro (2004).

Este artigo contém, além desta introdução, mais três seções. Na próxima, discutem-se os métodos utilizados no estudo; em seguida, os resultados são apresentados e algumas discussões são realizadas; na quarta e última, 
são apresentadas as principais conclusões obtidas da análise dos resultados.

\section{Metodologia}

Considerando-se que a agricultura é um setor importante para a geração de saldo positivo na balança comercial brasileira, sua modernização constitui fator necessário, dado seu potencial gerador de divisas para o país. A aferição da magnitude desse processo na região Norte foi feita pelo uso da análise fatorial aplicada a um conjunto de variáveis relacionadas com esse problema. Essa técnica permitiu quantificar a modernização agrícola na região e identificar quais indicadores estão associados a maior ou menor grau de modernização, o que foi possível por meio da construção de índices parciais e totais de modernização.

O Índice Relativo de Modernização Agrícola (IRMA) foi utilizado na medição da proporção de modernização da área de determinado município. Para isso, a construção do IRMA foi feita em duas etapas. Na primeira, desenvolveu-se o Indicador Parcial de Modernização Agrícola (IPMA), por meio de análise fatorial. Na segunda, com base no IPMA, foram estimados os pesos atribuídos a cada uma das variáveis que entraram na composição do IRMA, utilizando-se a análise de regressão.

\subsection{Análise fatorial}

A construção do indicador e do índice de modernização dos municípios da região Norte foi feita com base na análise fatorial por componentes principais. A análise fatorial é entendida como um conjunto de técnicas estatísticas que objetivam representar expressivo número de variáveis, de forma reduzida, como enfatizado por Kim e Mueller (1978). O método pauta-se na determinação das relações quantitativas entre as variáveis, associando àquelas com padrão semelhante o efeito de um fator causal subjacente e específico (Schilderinck, 1970). 
O modelo de análise fatorial é apresentado, genericamente, em forma matricial:

$$
X=\mu+\alpha f+\varepsilon,
$$

em que $X=\left(X_{1}, X_{2}, \ldots, X_{p}\right)^{\mathrm{t}}$ é um vetor transposto de variáveis aleatórias observáveis; $f=\left(f_{l}, f_{2}, \ldots, f_{r}\right)^{t}$ é um vetor transposto $(r<p)$ de variáveis não-observáveis ou fatores; $\alpha$, matriz ( $p \times x$ ) de coeficientes fixos ou cargas fatoriais; $\varepsilon=\left(\varepsilon_{1}, \varepsilon_{2}, \ldots, \varepsilon_{p}\right)^{t}$, vetor transposto de erros aleatórios.

A análise fatorial possui propriedades importantes. A primeira é que $\mathrm{E}(\varepsilon)=\mathrm{E}(\mathrm{f})=0$, e a segunda se refere aos fatores, que devem ser ortogonais. Nem sempre a estrutura inicial das estimativas das cargas fatoriais é definitiva. Com vistas em melhorar a interpretação dos fatores, o método proporciona a possibilidade de se fazer sua rotação. No caso, utilizou-se o método Varimax, de rotação ortogonal dos fatores ${ }^{4}$.

A estimação dos escores associados aos fatores obtidos, após a rotação ortogonal da estrutura fatorial inicial, situa cada observação no espaço dos fatores comuns. Logo, para cada fator $f_{i,}$ o $i$-ésimo escore fatorial a ser extraído é definido por $F_{i}$ e expresso por

$$
F_{i}=\sum_{i=1}^{n} b_{j} X_{i j}, \quad \operatorname{com} j=1,2, \ldots, p,
$$

em que $b_{j}$ são os coeficientes de regressão e $X_{i j}$, as $p$ variáveis observáveis.

Para estimar a variável $F_{i}$, que não é observável, utiliza-se a técnica de análise fatorial por meio da matriz $X$ de variáveis observáveis. A forma matricial a ser utilizada é a equação (2), devidamente reestruturada:

\footnotetext{
4 Maiores detalhes sobre o assunto podem ser encontrados em Dillon e Goldstein (1984); Johnson e Wichern (1988); e Basilevsky (1994).
} 
$F_{(n \times q)}=X_{(n \times p)} \cdot B_{(p \times q)}$

Os escores fatoriais são afetados pelas unidades em que as variáveis $\mathrm{X}_{\mathrm{i}}$ são medidas, tornando-se conveniente trabalhar com variáveis normalizadas. Dessa forma, substitui-se a variável $X_{i}$ pela variável normalizada $Z_{i j}$, expressando em desvios-padrão os desvios das observações originais em relação à sua média:

$\mathrm{Z}_{\mathrm{ij}}=\left[\left(\mathrm{X}_{\mathrm{i}}-\mu_{\mathrm{xi}}\right) / \sigma_{\mathrm{xi}}\right]$,

em que $\mu_{x i}$ é a média de $X_{i}$ e $\sigma_{x i}$ o seu desvio-padrão.

A equação (3) é então modificada, sendo reescrita da seguinte forma:

$F_{(n x q)}=Z_{(n x p)} \cdot \beta_{(p x q)}$.

Como as variáveis estão normalizadas em ambos os lados da equação, o vetor dos coeficientes de regressão $B$ é substituído pelo vetor $\beta$. Multiplicando-se os dois lados da equação (4) por $(1 / n) Z^{t}$, obtém-se:

$$
(1 / \mathrm{n}) Z^{\mathrm{t}} \mathrm{F}=(1 / \mathrm{n}) Z^{\mathrm{t}} \mathrm{Z} \beta
$$

em que n é o número de observações e $Z^{t}$, a matriz transposta de $Z$.

O primeiro membro da equação (5), (1/n) $Z^{t} \mathrm{~F}$, é a matriz de correlação entre os termos de $X_{i}$, que, a partir de agora, será representada por $R$. Já a matriz $(1 / \mathrm{n}) \mathrm{Z}^{\mathrm{t}} \mathrm{Zb}$ representa a correlação existente entre os escores fatoriais e os próprios fatores e será identificada por $\Lambda$. Assim, pode-se reescrever a equação (5), da seguinte forma:

$\Lambda=R \beta$.

Ao supor que a matriz $R$ seja não-singular, em que $|\mathrm{R}| \neq 0$, multiplicandose ambos os lados de (6) por $\left(R^{-1}\right)$, que é a inversa de $R$, tem-se: 
$\beta=R^{-1} . \Lambda$

Estimado o vetor $\beta$, pode-se substituí-lo na equação (4), para obter os escores fatoriais de cada observação.

\subsubsection{Construção do IPMA}

A propriedade de ortogonalidade dos escores fatoriais estimados foi utilizada na elaboração do IPMA. Entretanto, deve-se observar que a ortogonalidade associada à matriz de fatores não implica, necessariamente, a ortogonalidade dos escores fatoriais, devendo-se testar se os escores fatoriais são ortogonais por meio da matriz de variância e covariância entre esses escores.

A equação (8), usada para estimar o IPMA, pode ser expressa por

$$
\operatorname{IPMA}_{i}=\sqrt{\sum_{i=1}^{n} F_{i j}^{2}}, \quad \text { com j }=1,2, \ldots, p \text {, }
$$

em que IPMA é o indicador parcial de modernização associado ao $i$ ésimo município da região Norte; $\mathrm{F}_{\mathrm{ij}}$ são os escores fatoriais estimados, conforme procedimento de componentes principais.

Espera-se que os escores associados aos municípios tenham distribuição simétrica em torno da média zero. Assim, metade deles apresentará sinais negativos e a outra, sinais positivos, de modo que os municípios com menores índices de modernização parcial apresentarão escores fatoriais negativos. Com vistas em evitar que altos escores fatoriais negativos elevem a magnitude dos índices associados a esses municípios, é conveniente inseri-los no primeiro quadrante, conforme transformação: 


$$
\mathrm{F}_{\mathrm{ij}}=\frac{\left(\mathrm{F}-\mathrm{F}_{\text {min }}\right)}{\left(\mathrm{F}_{\text {max }}-\mathrm{F}_{\text {min }}\right)},
$$

em que $F_{\text {min }}$ e $F_{\text {max }}$ são os valores máximo e mínimo observados para os escores fatoriais associados aos municípios do Norte.

Por meio desse procedimento, consegue-se alocar todos os escores fatoriais no intervalo fechado entre zero e um. O cálculo geométrico do índice parcial de modernização é mostrado na Figura 1.

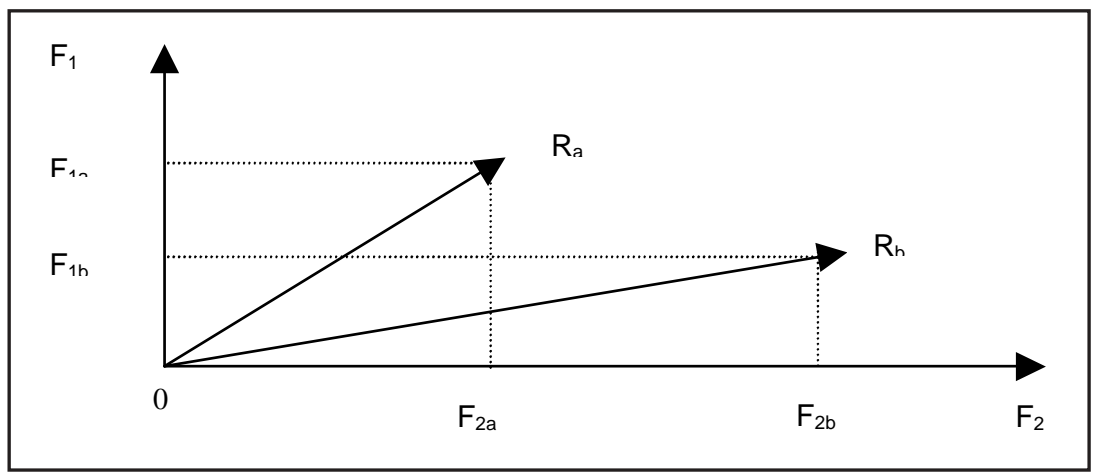

Figura 1 - Construção geométrica do IPMA.

Na Figura 1, observa-se que, associados ao município $A$, estão os escores fatoriais $F_{1 a}$ e $F_{2 a}$, em que o vetor IPMA $A_{a}$ é a resultante associada a esses escores fatoriais ortogonais, assim definidos:

$I P M A_{a}=\left(F_{1 a}^{2}+F_{2 a}^{2}\right)^{1 / 2}$.

Procedimento semelhante foi utilizado no município $B$. O tamanho da resultante $R_{a}$ ou $R_{b}$, respectivamente, determinará a magnitude do IPMA associado aos municípios A e B. Ressalta-se que o IPMA, definido dessa forma, é útil para fazer a hierarquização dos municípios do Norte, quanto ao nível de modernização; todavia, não serve para estimar o percentual de modernização de cada um dos municípios. Para tal, utiliza-se o IRMA. 


\subsubsection{Construção do IRMA}

$\mathrm{Na}$ construção do $\mathrm{IRMA}_{\mathrm{i}}$, associado ao i-ésimo município da região Norte, definiu-se a equação:

$$
\operatorname{IRMA}_{\mathrm{i}}=1-\left(\sum_{\mathrm{i}=1}^{\mathrm{n}} \mathrm{P}_{\mathrm{j}} \mathrm{X}_{\mathrm{i}}\right) \text {, }
$$

em que os pesos $P_{j}$ são estimados por regressão múltipla; a variável dependente é o IPMA ; e as variáveis explicativas são os indicadores utilizados na construção do IRMA, sendo o modelo econométrico dado por (12) estimado para determinar os pesos associados aos indicadores utilizados.

$$
\text { IPMA }_{i}=\alpha+\beta_{1} \text { DIX } 1+\beta_{2} \text { DIX } 2+\beta_{3} \text { DIX } 3 \ldots \beta_{22} \text { DIX } 22+\varepsilon_{i} \text {, }
$$

em que IPMA ${ }_{i}$ corresponde ao indicador de modernização agrícola para o i ésimo município da região Norte, $\alpha$ e os $\beta$ 's são parâmetros desconhecidos a serem estimados pelo Método dos Mínimos Quadrados Ordinários, DIX1...DIX22 correspondem aos indicadores utilizados e $\varepsilon_{i}$, ao termo de erro com as pressuposições usuais.

\subsubsection{Indicadores de modernização}

A modernização agrícola, nesta análise, está relacionada com indicadores que sugerem progresso econômico, tecnológico e social. Nesse sentido, é um processo associado à maior utilização de tratores, irrigação, investimentos, financiamentos, etc.

Em face do exposto, a construção do IRMA levou em consideração vinte e dois indicadores. Por um lado, aqueles relativos às despesas com insumos diversos e aos investimentos em instalações visam incorporar o crescente gasto com insumos e capitalização da agricultura; por outro, os relativos aos financiamentos, que são os que têm maior parte da responsabilidade pelas transformações ocorridas na agricultura. 
Em relação à tecnologia, pretende-se determinar a intensidade de seu uso, e não apenas o seu volume, razão pela qual a maioria das variáveis utilizadas está expressa em mão-de-obra $(\mathrm{EH})$ ou em área explorada (AE). O conceito de área explorada, segundo Hoffman (1992), engloba as atividades admitidas na área trabalhada e as áreas cobertas por pastagens e matas naturais.

A construção de um índice de modernização envolve um conhecimento, a priori, de quais seriam os níveis ideais de modernização associados aos indicadores que são utilizados na sua construção. Essa tarefa seria extremamente difícil, visto que poderiam ocorrer variações, de acordo com quem estivesse fazendo a análise.

No critério de hierarquização, tomou por base os municípios que apresentavam melhor posicionamento em cada um dos indicadores empregados para aferir a modernização. Em 1995, havia 398 municípios na região Norte, dois quais foram consideradas as médias que serviram de base para estimação da média aritmética de cada indicador, cujos valores foram tomados como referência de modernização. Isso significa dizer que, quanto mais distante estiver o valor encontrado de um município em relação à média estimada de determinado indicador, menor a sua modernização.

Levando-se em consideração o exposto, foram selecionados os indicadores de acordo com diversos trabalhos que abordaram a modernização agrícola, incluindo-se um indicador adicional, o IDH, que não é freqüentemente utilizado nesses casos.

IX1 Número de tratores/AE;

IX1 $1_{\text {ref }}$ Média do IX1;

IX2 Número de tratores/EH;

IX2 ${ }_{\text {ref }}$ Média do IX2;

IX3 Número de estabelecimento com controle de pragas e doenças/AE; IX3 ${ }_{\text {ref }}$ Média do IX3;

IX4 Número de estabelecimento que aplicam adubos e corretivos/TE; IX $4_{\text {ref }}$ Média do IX4; 
IX5 Número de estabelecimento com eletricidade/TE;

IX5 ${ }_{\text {ref }}$ Média do IX5;

IX6 Quantidade de eletricidade consumida/AE;

IX6 ${ }_{\text {ref }}$ Média do IX6;

IX7 Área irrigada/AE;

IX7 ${ }_{\text {ref }}$ Média do IX7;

IX8 Combustíveis e lubrificantes/AE;

IX8 ${ }_{\text {ref }}$ Média do IX8;

IX9 EH/AE;

IX9 ${ }_{\text {ref }}$ Média do IX9;

IX10 Número de estabelecimento com assistência técnica/AE;

IX10 ${ }_{\text {ref }}$ Média do IX10;

IX11 Valor de financiamentos/AE;

IX11 ${ }_{\text {ref }}$ Média do IX11;

IX12 Valor de investimentos/AE;

IX12 ref Média do IX12;

IX13 Valor de financiamentos/EH;

IX13 ref Média do IX13;

IX14 Valor de investimentos/EH;

IX14 ${ }_{\text {ref }}$ Média do IX14;

IX15 Valor da produção/AE.

IX15 ref Média do IX15;

IX16 Valor da produção/EH;

IX16 ${ }_{\text {ref }}$ Média do IX16;

IX17 Despesas/AE;

IX17 ref Média do IX17;

IX18 Despesas/EH;

IX18 ${ }_{\text {ref }}$ Média do IX18;

IX19 Número de estabelecimento com controle de pragas e doenças/EH;

IX19 ${ }_{\text {ref }}$ Média do IX19;

IX20 Número de estabelecimento com assistência técnica/EH;

IX20 ref Média do IX20;

IX21 Área irrigada/TE;

IX21 ${ }_{\text {ref }}$ Média do IX21;

IX22 Índice de Desenvolvimento Humano (IDH); 


\section{IX22 ${ }_{\text {ref }}$ Média do IX22;}

sendo AE a Área explorada; EH, Equivalente-homem; e TE, Total de estabelecimentos agrícolas.

Com base nos indicadores supracitados, passou-se a estruturar aqueles que entram na construção do IPMA e do IRMA, cujas definições são as seguintes:

DIX1 $=0$, quando IX1 $\geq$ IX1 $_{\text {ref }} ;$ e igual a $\left[1-\left(\right.\right.$ IX1 IX1 $\left.{ }_{\text {ref }}\right)$ *100, nos demais casos; DIX2 $=0$, quando IX2 IX $2_{\text {ref }}$ e igual a $\left[1-\left(\mathrm{IX} 2 \mathrm{IX} 2_{\text {ref }}\right)\right] * 100$, nos demais casos;

DIX22 $=0$, quando IX22 IX22 $2_{\text {ref }}$; igual a $\left[1-\left(\right.\right.$ IX22 IX22 $\left.2_{\text {ref }}\right) * 100$, nos demais casos.

Observa-se que tanto o IPMA como o IRMA correspondem a um indicador e um índice relativo de modernização, que tomam como referência a média aritmética dos indicadores municipais.

\subsection{Fonte de dados}

Os dados utilizados neste trabalho são provenientes do Censo Agropecuário 1995/96, obtidos no site do Instituto Brasileiro de Geografia e Estatística - IBGE.

\section{Resultados e discussões}

Dado que o interesse da pesquisa recai sobre a aferição da modernização agrícola da região Norte, é conduzida, inicialmente, uma análise fatorial que agrega as observações feitas sobre os vinte e dois indicadores considerados.

No intuito de verificar se os dados suportam uma análise fatorial, são realizados alguns testes estatísticos. Um deles é o teste de esfericidade de Bartlett, cujo objetivo é constatar a presença de correlações entre as variáveis. Após a realização do teste, que atingiu valor igual a 5.403,442, verificou-se a sua significância a $1 \%$ de probabilidade, resultado que permite rejeitar a hipótese nula de que a matriz de correlação seja uma 
matriz identidade.

Outra forma de quantificar o grau de intercorrelações das variáveis e a adequadabilidade da análise fatorial ao conjunto de dados é a medida de adequação da amostra, a qual pode ter valores de 0 a 1, atingindo a unidade quando cada variável for perfeitamente predita pelas demais. $\mathrm{Na}$ tentativa de medir essa adequabilidade, este estudo utiliza o teste de Kaiser-Meyer-Olkin (KMO), cujo valor obtido foi de 0,83. Conforme a classificação fornecida por HAIR et al. (1995), valores acima de 0,5 indicam que os dados são adequados à realização da análise fatorial. Por meio dos testes, conclui-se que as variáveis utilizadas estão de acordo com o procedimento da análise fatorial, o que possibilita a continuidade deste estudo. O emprego do método de componentes principais gerou seis fatores com raízes características maiores que um (Tabela 1).

Tabela 1 - Fatores obtidos pelo método dos componentes principais

Fonte: Resultados da pesquisa.

Constata-se que a contribuição acumulada dos fatores 1 a 6, para explicação da variância total dos indicadores utilizados, é de 77,97\%. Esses valores mostram que a utilização de seis fatores é suficiente à análise.

É importante ressaltar que pelo menos dois motivos explicam a existência de seis raízes características na análise. O primeiro se refere à baixa 
correlação entre os indicadores utilizados na análise fatorial, enquanto o segundo diz respeito à grande heterogeneidade, quanto à modernização, presente na região Norte.

Para melhor interpretação dos dados, os fatores foram submetidos a uma rotação ortogonal pelo método Varimax. Segundo Kim e Mueller (1978), essa rotação altera a contribuição de cada fator para a variância, sem, contudo, modificar a contribuição conjunta destes. A principal vantagem da rotação é permitir que os novos fatores se relacionem, claramente, com determinados grupos de variáveis, facilitando a análise da solução encontrada. A Tabela 2 determina quais fatores se relacionam com quais variáveis, ao exibir as cargas fatoriais, as comunalidades e o percentual da variância total dos indicadores. 
Tabela 2 - Cargas fatoriais e comunalidades, após a rotação ortogonal pelo método varimax

\begin{tabular}{|c|c|c|c|c|c|c|c|}
\hline \multirow{2}{*}{ INDICADOR } & \multicolumn{6}{|c|}{ CARGA FATORIAL } & \multirow{2}{*}{ COMUNALIDADE } \\
\hline & $\mathrm{F} 1$ & $\mathrm{~F} 2$ & F3 & $\mathrm{F} 4$ & F5 & F6 & \\
\hline INDX1 & 0,3860 & $(0,1980)$ & 0,0189 & 0,8310 & 0,1630 & 0,0706 & 0,9107 \\
\hline INDX2 & 0,1460 & 0,0704 & $(0,0850)$ & 0,9210 & 0,0487 & 0,0622 & 0,8880 \\
\hline INDX3 & 0,4370 & $(0,1150)$ & $(0,2730)$ & 0,2790 & 0,6790 & $(0,0392)$ & 0,8191 \\
\hline INDX4 & 0,0094 & 0,3370 & 0,4840 & 0,2120 & 0,4170 & 0,3630 & 0,6985 \\
\hline INDX5 & $(0,1090)$ & 0,6740 & 0,1540 & $(0,0586)$ & 0,3230 & 0,2150 & 0,6439 \\
\hline INDX6 & $(0,3090)$ & 0,1500 & 0,8110 & $(0,1740)$ & $(0,1450)$ & 0,0602 & 0,8306 \\
\hline INDX7 & 0,2200 & 0,1600 & $(0,2900)$ & 0,2180 & 0,2910 & 0,6380 & 0,6973 \\
\hline INDX8 & 0,8340 & $(0,0603)$ & $(0,2740)$ & 0,2040 & 0,1550 & $(0,0206)$ & 0,8403 \\
\hline INDX9 & $\mathbf{0 , 8 5 0 0}$ & $(0,4040)$ & $(0,0806)$ & 0,1850 & 0,0995 & $(0,0108)$ & 0,9364 \\
\hline INDX10 & 0,6890 & $(0,1050)$ & $(0,0791)$ & $(0,0530)$ & 0,5830 & 0,0527 & 0,8375 \\
\hline INDX11 & 0,3240 & 0,1120 & $(0,1630)$ & 0,3260 & 0,5230 & $(0,0910)$ & 0,5322 \\
\hline INDX12 & $(0,0870)$ & 0,3550 & 0,8150 & $(0,0210)$ & $(0,0020)$ & 0,0036 & 0,7983 \\
\hline INDX13 & $(0,4400)$ & 0,4420 & 0,6110 & $(0,0999)$ & $(0,0725)$ & 0,0061 & 0,7776 \\
\hline INDX14 & $(0,4610)$ & 0,7180 & 0,2430 & $(0,0567)$ & 0,1020 & 0,0424 & 0,8025 \\
\hline INDX15 & 0,9150 & $(0,2050)$ & $(0,1530)$ & 0,1800 & 0,0765 & $(0,0273)$ & 0,9417 \\
\hline INDX16 & 0,0150 & 0,8950 & 0,0733 & $(0,0080)$ & $(0,1670)$ & 0,0649 & 0,8388 \\
\hline INDX17 & 0,8340 & $(0,0118)$ & $(0,1650)$ & 0,3150 & 0,2290 & $(0,0083)$ & 0,8747 \\
\hline INDX18 & $(0,2930)$ & 0,8470 & 0,2450 & 0,0610 & 0,0177 & 0,1430 & 0,8878 \\
\hline INDX19 & $(0,6560)$ & 0,4370 & 0,1860 & $(0,0401)$ & 0,4250 & $(0,0306)$ & 0,8391 \\
\hline INDX20 & $(0,0944)$ & 0,4760 & 0,2640 & $(0,3190)$ & 0,4800 & 0,2350 & 0,6926 \\
\hline INDX21 & $(0,0844)$ & 0,2500 & 0,2230 & $(0,0562)$ & $(0,1180)$ & 0,7900 & 0,7605 \\
\hline INDX22 & $(0,2890)$ & $(0,1190)$ & 0,3200 & 0,0641 & $(0,1090)$ & 0,2960 & 0,3037 \\
\hline \% da Variância & 23,0840 & 17,0690 & 12,1200 & 9,9490 & 9,3600 & 6,3900 & - \\
\hline
\end{tabular}

Fonte: Resultados da pesquisa.

A contribuição dos seis fatores ${ }^{5}$ para a variância foi modificada após a rotação, como pode ser observado na última linha da Tabela 2. Entretanto, deve-se destacar que a variação total permaneceu constante e igual a $77,97 \%$. Para fins de interpretação, as cargas fatoriais acima de 0,6 estão em negrito, com vistas em evidenciar os indicadores mais fortemente associados a determinado fator.

${ }^{5}$ No cálculo do IPMA foram utilizados apenas dois fatores, em razão de estes terem apresentado melhor ajuste. 
Pode-se constatar que o fator 1 se encontra fortemente correlacionado com os indicadores INDX8 (combustíveis e lubrificantes/AE), INDX9 (EH/AE), INDX10 (número de estabelecimentos com assistência técnica/ AE), INDX15 (valor da produção/AE), INDX17 (despesas/AE), INDX19 (número de estabelecimentos com controle de pragas e doenças/EH); o fator 2 está mais correlacionado com os indicadores INDX5 (número de estabelecimentos com eletricidade/AE), INDX14 (valor de investimento/ EH), INDX16 (valor da produção/EH) e INDX18 (despesas/EH); o fator 3, com os fatores INDX6 (quantidade de energia elétrica/AE), INDX12 (valor do investimento/AE) e INDX13 (valor de financiamento/EH); o fator 4, com INDX1 (número de tratores/AE) e INDX2 (número de tratores/EH); e o fator 5, com INDX3 (número de estabelecimento com controle de pragas e doenças/AE); por fim, o fator 6 está mais correlacionado com INDX7 (área irrigada/AE) e com INDX21 (área irrigada/TE).

Com isso, podem-se denominar F1, F2, F3, F4, F5 e F6 de, respectivamente, intensificação do trabalho e da produtividade por área explorada; intensificação da utilização de fatores econômicos e intensificação da utilização de investimento e energia elétrica em relação equivalente-homem; intensificação da utilização de fatores econômicos e intensificação da utilização de investimento e energia elétrica em relação à área explorada; utilização de tratores; controle de pragas e doenças; e irrigação.

Após obtenção dos fatores e coeficientes (cargas fatoriais) necessários à estimação dos escores fatoriais, calculou-se o IPMA de cada município da região Norte. Estimado o IPMA e encontrados os pesos associados a cada um dos indicadores obtidos por meio de uma análise de regressão linear, em que o IPMA foi a variável dependente e os indicadores INDX1 a INDX22, variáveis independentes, foram então estimados os respectivos IRMA $_{\mathrm{s}}$. Deve-se destacar que os resultados ao longo do trabalho, por motivo de espaço, são apresentados apenas para os estados, e só alguns deles são discutidos. 
A região Norte possui um índice médio de modernização igual a 34,30\% (Tabela 3), valor bastante razoável, dada a dimensão dessa região e dadas as suas condições de infra-estrutura. Entretanto, deve-se ressaltar que os valores máximo e mínimo do IRMA variam significativamente. Enquanto há apenas um município com IRMA acima de $90 \%$, que é o de Barcelos no Amazonas, no extremo oposto existem 154 municípios com IRMA inferior a 20\%. Esses resultados mostram a discrepância existente entre os municípios, o que evidencia a convivência de sistemas com alta e baixa modernização agrícola na região.

Tabela 3 - Estatísticas gerais do IRMA da região Norte, 1995/1996

\begin{tabular}{lc}
\hline \multicolumn{1}{c}{ ESTATÍSTICA } & IRMA \% \\
\hline MÁXIMO & 94,10 \\
MÉDIA & 34,30 \\
MÍNIMO & 0,01 \\
DESVIO & 22,49 \\
VARIÂNCIA & 05,06 \\
MEDIANA & 26,00 \\
\hline
\end{tabular}

Fonte: Resultados da pesquisa.

Em relação aos estados, observa-se, Tabela 4, que o Acre é o que tem maior desigualdade em relação ao IRMA, visto que seu desvio-padrão representa 68,29\% da média. Este resultado é acompanhado pelo Pará e por Tocantins, onde os desvios representam, respectivamente, 57,09 e $47,24 \%$ da média. Claramente, isto é indicador da grande desigualdade regional. Roraima e Amazonas destacam-se por apresentar menores níveis de heterogeneidade em relação aos demais estados; contudo, vale destacar que, mesmo sendo um grau de heterogeneidade relativamente inferior, continuam indicando alto grau de diferenças em relação ao IRMA. 
Tabela 4 - Estatísticas gerais do IRMA, por estados, na região Norte, 1995/1996

Fonte: Resultados da pesquisa.

Com o intuito de classificar os estados em níveis de modernização, optouse pela utilização de cinco conceitos, os quais são representados pelas letras A, B, C, D e E, obedecendo-se ao seguinte critério: de $0 \%$ a $20 \%$ - "E"; de $21 \%$ a $40 \%$ - "D"; de $41 \%$ a $60 \%$ - "C"; de $61 \%$ a $80 \%$ - "B" e de $81 \%$ a $100 \%$ - "A". Após esta classificação, tabularam-se os conceitos por estado, conforme Tabela 5. Com isso, é possível observar, de forma clara, uma grande heterogeneidade existente no IRMA.

\begin{tabular}{cccccccc}
\hline ESTATÍSTICAS & ACRE & AMAZONAS & AMAPÁ & RORAIMA & RONDÔNIA & PARÁ & TOCANTINS \\
\hline MÁXIMO & 86,31 & 94,10 & 71,86 & 36,22 & 57,23 & 88,00 & 30,83 \\
MÉDIO & 30,28 & 58,75 & 44,13 & 23,99 & 29,22 & 40,44 & 17,42 \\
MÍNIMO & 7,80 & 14,22 & 21,07 & 8,74 & 6,44 & 2,24 & 0,01 \\
DESVIO-PADRÃO & 20,68 & 20,04 & 17,72 & 7,76 & 12,29 & 23,09 & 8,23 \\
\hline
\end{tabular}


Tabela 5 - IRMA por estados e conceitos da região Norte

\begin{tabular}{|c|c|c|c|c|c|c|}
\hline ESTADOS & $\mathrm{A}$ & B & $\mathrm{C}$ & $\mathrm{D}$ & $E$ & TOTAL \\
\hline \multirow{2}{*}{ ACRE } & 2,00 & - & 1,00 & 11,00 & 8,00 & 22,00 \\
\hline & $9,09 \%$ & $0,00 \%$ & $4,55 \%$ & $50,00 \%$ & $36,36 \%$ & $100,00 \%$ \\
\hline \multirow{2}{*}{ AMAZONAS } & 9 & 22 & 20 & 9 & 2 & 62 \\
\hline & $14,52 \%$ & $35,48 \%$ & $32,26 \%$ & $14,52 \%$ & $3,23 \%$ & $100,00 \%$ \\
\hline \multirow{2}{*}{ AMAPÁ } & - & 4 & 4 & 7 & - & 15 \\
\hline & $0,00 \%$ & $26,67 \%$ & $26,67 \%$ & $46,67 \%$ & $0,00 \%$ & $100,00 \%$ \\
\hline \multirow{2}{*}{ RORAIMA } & - & - & - & 7 & 1 & 8 \\
\hline & $0,00 \%$ & $0,00 \%$ & $0,00 \%$ & $87,50 \%$ & $12,50 \%$ & $100,00 \%$ \\
\hline \multirow{2}{*}{ RONDÔNIA } & - & - & 9 & 21 & 10 & 40 \\
\hline & $0,00 \%$ & $0,00 \%$ & $22,50 \%$ & $52,50 \%$ & $25,00 \%$ & $100,00 \%$ \\
\hline \multirow{2}{*}{ PARÁ } & 2 & 38 & 18 & - & 70 & 128 \\
\hline & $1,56 \%$ & $29,69 \%$ & $14,06 \%$ & $0,00 \%$ & $54,69 \%$ & $100,00 \%$ \\
\hline \multirow{2}{*}{ TOCANTINS } & - & - & - & 60 & 63 & 123 \\
\hline & $0,00 \%$ & $0,00 \%$ & $0,00 \%$ & $48,78 \%$ & $51,22 \%$ & $100,00 \%$ \\
\hline TOTAL & 13,00 & 64,00 & 52,00 & 115,00 & 154,00 & 398,00 \\
\hline$\%$ & $3,27 \%$ & $16,08 \%$ & $13,07 \%$ & $28,89 \%$ & $38,69 \%$ & $100,00 \%$ \\
\hline
\end{tabular}

Fonte: Resultados da pesquisa.

Em relação a cada estado, observa-se que Roraima destaca-se pelo baixo desvio-padrão, o que indica que, em relação à modernização agrícola, não existem grandes disparidades estaduais. Nesse sentido, é mister mencionar que Amazonas e Amapá seguem a mesma trajetória, enquanto Acre, Rondônia, Tocantins e Pará apresentam grandes disparidades, e o primeiro estado destaca-se dos demais.

No estado do Acre, há dois municípios que se encontram no conceito A, embora mais de $86 \%$ destes estejam em estratos de modernização inferiores. O Amazonas apresenta um perfil mais moderno, pois 50\% dos seus municípios estão com níveis de modernização superiores a $60 \%$, além de apenas $17,75 \%$ dos municípios apresentarem IRMA inferior a 
40\%. Em relação ao Amapá, observa-se que, aproximadamente, 73,50\% possuem IRMA inferior a $80 \%$, e quase $50 \%$ encontram-se em baixíssimo nível de modernização. Em adição, Roraima destaca-se por possuir baixíssimo IRMA. Resultado surpreendente ocorreu em Rondônia, já que apresentou um resultado surpreendente, pois apresentou baixa média do IRMA, já que este estado apresentou indicadores, na maioria das vezes, abaixo da média. O Pará, por sua vez, apresentou aspecto interessante, visto que teve valores extremos representados por A, B e E. Por fim, Tocantins não surpreendeu, pois é um estado relativamente novo, o que justifica seu baixo IRMA. Vale ressaltar que, em determinados municípios do Tocantins, a modernização agrícola não se faz presente, ou está presente em níveis extremamente baixos.

\section{Conclusão}

Os resultados indicam que, em relação à modernização agrícola, a região Norte apresenta-se bastante heterogênea, quando comparada às demais regiões. As diferenças estão presentes também na própria região, quando estados e municípios são confrontados. Observa-se que o Amazonas apresenta melhores resultados do IRMA, seguido pelo Amapá, ao contrário de Tocantins e Roraima, que obtiveram os piores valores.

De forma geral, observa-se que esta região apresenta baixos níveis estaduais de modernização, os quais se justificam pela recente expansão da fronteira agrícola, pelo baixo nível de investimentos governamentais, pela falta de políticas públicas voltadas ao desenvolvimento agrícola e, por fim, pelos movimentos ambientalistas.

A principal limitação deste trabalho reside na construção do IRMA. Verificou-se que, em alguns casos, municípios que apresentaram grande número de indicadores acima da média tiveram baixo IRMA. Esse fato motiva investigações futuras, com vistas em determinar o impacto dos indicadores no IPMA e no IRMA. Com isso, será possível determinar quais indicadores seriam mais eficientes na elaboração de políticas 
públicas que visem aumentar o grau de modernização de determinada região.

\section{Referências bibliográficas}

BASILEVSKY, A. Statististical Factor Analysis and Related Methods: Theory and Applications, New York, 1994.

DILLON, W.; GOLDSTEIN, M. Multivariate Analysis: Methods and Applications, New York, 1984.

FERNANDES, T.A.G.; LIMA, J.E. Uso de Análise Multivariada para Identificação de Sistemas de Produção, Brasília: Revista Pesquisa Agropecuária Brasileira, 26(10): 1823-1836, out, 1991.

FERNAU, M.E.; SAMSON, P.J. Use of cluster analysis to define periods of similar meteorology and precipitation hemistry in Eastern North America, Part I: Transport patterns, Journal of Applied Meteorology, Michigan, v. 29, p. 735-761, 1990.

FERREIRA Jr., S.; BAPTISTA, A.J.M.S. A modernização agropecuária nas microrregiões do Estado de Minas Gerais. Revista de Economia e Sociologia Rural, Brasília: SOBER, V. 42, No. 01, 2004.

GONG, X.; RICHMAN, M.B. On the application to growing season precipitation data in North America East of the rockies, Journal of Clirmate, Oklahoma, v. 8, p. 897-931, 1995.

HAIR, J.F.; ANDERSON, R.E.;TATHAM, R.L.; BLACK, W.C. Multivariate Data Analysis: With Readings, Prentice Hall, New Jersey, 1995.

HOFFMAN, R.. A dinâmica da modernização da agricultura em 157 microrregiões homogêneas do Brasil. Revista de Economia e Sociologia Rural, Brasília: SOBER, V. 30, No. 4. 
IBGE - Instituto Brasileiro de Geografia e Estatística. Censo Agropecuário, n. 3, Rio de Janeiro: IBGE, 1995.

JOHNSON, A.; WICHERN, D. Applied Multivariate Statistical Analysis, New Jersey, 1988.

LEMOS, J.J.S. Níveis de degradação no Nordeste Brasileiro. Revista Econômica do Nordeste. V. 32. n.3. pág. 406 - 429, 2001b

KIN, J.; MUELLER, C.W. Introduction to Factor Analysis: What It is and How to Do It, Sage Publications, London, 1978.

POLLAK, L.M.; CORBETT, J.D. Using GIS datasets to classify maizegrowing regions in Mexico and Central America, Agronomy Journal, v.85, p. 1133-1139, 1993.

SILVA, R.G.; RIBEIRO, C.G. Análise da degradação ambiental na Amazônia Ocidental: um estudo de caso dos municípios do Acre. Revista Brasileira de Economia Rural. V.42. n. 1, pág. 93 - 112.

SOUZA, B. M.; LIMA, J.E. Intensidade e dinâmica da modernização agrícola no Brasil e nas unidades de federação. Revista Brasileira de Economia. Rio de Janeiro: FGV. V.57, No. 4, pág. 795 - 824.

\footnotetext{
Abstract - The agricultural activity possesses expressive relevance for the agriculture of the North region. In relation to the agricultural modernization, the researches are driven in a joined way (national), the one that disables verify, indeed, which the degree of municipal development and which the variables that to impact this development. In a general way, it intends to determine the degree of agricultural modernization in the municipal districts of the North region. It is observed that this area presents low state levels of modernization, which are justified for the recent expansion of the agricultural border, for the low level of government investments, for the lack of public politics destined to the agricultural development and, finally, for the movements environmentalists.
}

Keywords: Agricultural modernization, north region and factorial analysis. 
REVISTA DE ECONOMIA E AGRONEGÓCIO, VOL.3, No 1 